\title{
Deficiency of Isoprenylcysteine Carboxyl Methyltransferase (ICMT) Leads to Progressive Loss of Photoreceptor Function
}

\author{
Jeffrey R. Christiansen, ${ }^{1 \star}$ Nachiket D. Pendse, ${ }^{1,2,5 \star}$ Saravanan Kolandaivelu, ${ }^{1}$ Martin 0. Bergo, ${ }^{3}$ Stephen G. Young, ${ }^{4}$ \\ and Visvanathan Ramamurthy ${ }^{1,2,5}$ \\ Departments of ${ }^{1}$ Ophthalmology, ${ }^{2}$ Biology, and ${ }^{5}$ Biochemistry, West Virginia University, Morgantown, West Virginia 26506, ${ }^{3}$ Sahlgrenska Cancer Center, \\ University of Gothenburg, Gothenburg 405 30, Sweden, and ${ }^{4}$ Departments of Medicine and Human Genetics, University of California-Los Angeles, \\ Los Angeles, California 90095
}

Retinal neurons use multiple strategies to fine-tune visual signal transduction, including post-translational modifications of proteins, such as addition of an isoprenyl lipid to a carboxyl-terminal cysteine in proteins that terminate with a "CAAX motif." We previously showed that RAS converting enzyme 1 (RCE1)-mediated processing of isoprenylated proteins is required for photoreceptor maintenance and function. However, it is not yet known whether the requirement for the RCE1-mediated protein processing is related to the absence of the endoproteolytic processing step, the absence of the subsequent methylation step by isoprenylcysteine methyltransferase (ICMT), or both. To approach this issue and to understand the significance of protein methylation, we generated mice lacking Icmt expression in the retina. In the absence of Icmt expression, rod and cone light-mediated responses diminished progressively. Lack of ICMT-mediated methylation led to defective association of isoprenylated transducin and cone phosphodiesterase 6 (PDE6 $\alpha^{\prime}$ ) with photoreceptor membranes and resulted in decreased levels of transducin, PDE6 $\alpha^{\prime}$, and cone G-protein coupled receptor kinase-1 (GRK1). In contrast to our earlier findings with retina-specific Rce1 knock-out mice, rod PDE6 in Icmt-deficient mice trafficked normally to the photoreceptor outer segment, suggesting that the failure to remove the $-A A X$ is responsible for blocking the movement of PDE6 to the outer segment. Our findings demonstrate that carboxyl methylation of isoprenylated proteins is crucial for maintenance of photoreceptor function.

Key words: electroretinogram; phototransduction cascade; post-translational protein modification; protein methylation; protein stability; retinal neurons

\section{Significance Statement}

In this report, we show that an absence of isoprenylcysteine methyltransferase-mediated protein methylation leads to progressive loss of vision. Photoreceptors also degenerate, although at a slower pace than the rate of visual loss. The reduction in photoresponses is due to defective association of crucial players in phototransduction cascade. Unlike the situation with RCE1 deficiency, where both methylation and removal of $-A A X$ were affected, the transport of isoprenylated proteins in isoprenylcysteine methyltransferase-deficient retinas was not dependent on methylation. This finding implies that the retention of the $-A A X$ in PDE6 catalytic subunits in $\mathrm{Rce}^{-1-}$ mice is responsible for impeding their transport to the rod photoreceptor outer segment. In conclusion, lack of methylation of isoprenylcysteines leads to age-dependent photoreceptor dysfunction.

\section{Introduction}

Prenylation refers to the addition of a 15-carbon farnesyl or a 20-carbon geranylgeranyl lipid to the C-terminal cysteine of pro-

Received Jan. 13, 2016; revised March 18, 2016; accepted March 25, 2016.

Author contributions: J.R.C., N.D.P., S.K., and V.R. designed research; J.R.C., N.D.P., and S.K. performed research; M.O.B. and S.G.Y. contributed unpublished reagents/analytic tools; J.R.C., N.D.P., S.K., and V.R. analyzed data; J.R.C., N.D.P., M.O.B., S.G.Y., and V.R. wrote the paper.

This work was supported by National Institutes of Health Grants R01EY017035 to V.R. and R01HL126551 to S.G.Y., West Virginia Lions, and West Virginia University Unrestricted Research to Prevent Blindness Challenge Grant. We thank Thamarai Saravanan for maintaining animal stocks; Dr. Karen Martin for advice and the use of the West Virginia University Microscopic Imaging Facility; and members of the V.R. laboratory and Dr. Neal Peachey at Cleveland Clinic for constructive criticism. teins ending in a "CAAX" motif, where $C$ refers to a cysteine, $A$ for an aliphatic amino acid, and $X$ for any amino acid (Svensson et al., 2006) (Fig. 1, Step 1). After prenylation, the last three amino acid residues $(-A A X)$ are cleaved by RAS-converting enzyme 1

The authors declare no competing financial interests.

*J.R.C. and N.D.P. contributed equally to this work.

Correspondence should be addressed to Dr. Visvanathan Ramamurthy, Department of Ophthalmology, One Medical Center Drive, West Virginia University, Morgantown, WV 26506-9193. E-mail: ramamurthyv@wvumedicine.org.

J.R. Christiansen's present address: Prion Research Center at Colorado State University, Fort Collins, Colorado 80523.

DOI:10.1523/JNEUROSCI.0176-16.2016

Copyright $\odot 2016$ the authors $\quad 0270-6474 / 16 / 365107-08 \$ 15.00 / 0$ 


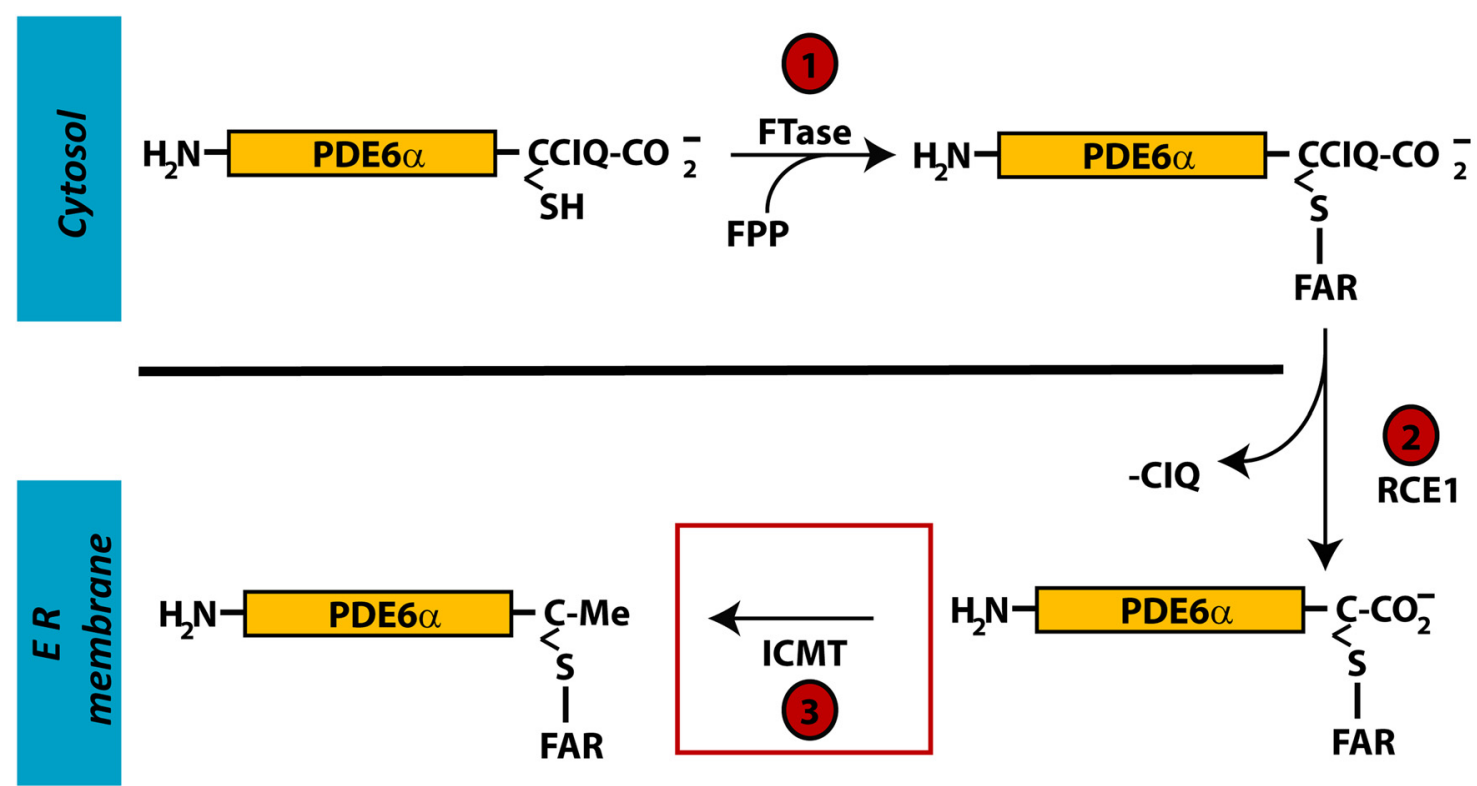

Figure 1. Schematic representation of prenylation and postprenylation processing. Rod phosphodiesterase6 (PDE6 $\alpha$ ) is farnesylated. Step 1, Protein farnesyltransferase (FTase-I), a cytosolic protein, adds a farnesyl lipid (FPP) to the cysteine of the CAAX (CAAX = CCIQ for PDE6 $\alpha$ ) motif. Step 2, RCE1-mediated endoproteolysis at the ER membrane cleaves the last three amino acids of the CCIQ motif (i.e., - CIQ).Step 3, ICMT, an ER membrane protein, catalyzes the methyl esterification of the farnesylcysteine. After methylation, prenylated proteins are extracted from ER membranes by proteins, such as PrBP $\delta$, and further transported to photoreceptor 0 .

(RCE1) at the endoplasmic reticulum (ER) membrane (Fig. 1, Step 2). The final event of "CAAX protein" processing is methyl esterification of the newly exposed isoprenyl cysteine by ICMT, an integral ER membrane protein that uses $S$-adenosyl methionine (SAM) as the methyl donor (Fig. 1, Step 3) (Hrycyna et al., 1991; Griggs et al., 2010).

Experiments designed to identify methylated photoreceptor proteins uncovered a subset of rod outer segment (OS) proteins that incorporated a radioactive methyl group (Swanson and Applebury, 1983). The identity of the methylated proteins and their ability to incorporate a methyl group have been studied extensively, but the importance of this modification to photoreceptor function is not known (Ong et al., 1989; Ohguro et al., 1991; Inglese et al., 1992). Rod phosphodiesterase 6 (PDE6), the effector enzyme of the visual signal transduction cascade, was the first methylated protein to be identified in retinal lysates (Swanson and Applebury, 1983). The carboxyl terminus of PDE6 catalytic subunits terminates with a so-called "CAAX motif," which triggers isoprenylation of the carboxyl-terminal cysteine (the " $C$ " of the CAAX motif). PDE6 $\alpha$ and $\beta$ catalytic subunits are isoprenylated by farnesyl and geranylgeranyl lipids, respectively. Interestingly, PDE6 $\alpha$ incorporated a methyl group in an in vitro radioactive methylation assay, whereas PDE6 $\beta$ was not an efficient substrate for protein methylation (Anant et al., 1992). The methylation status of PDE6 $\alpha^{\prime}$, which is thought to be geranylgeranylated, is not known. Additional isoprenylated (farnesylated) photoreceptor proteins include the $\gamma$-subunit of transducin $(\mathrm{G} \gamma \mathrm{T} 1)$ and rhodopsin kinase (GRK1) (Fukada et al., 1990; Inglese et al., 1992).

Methylation of isoprenylated cysteines is thought to increase the hydrophobicity of the protein and facilitate interactions with membranes (Parish and Rando, 1996). Also, in vitro approaches have shown that carboxyl methylation enhances certain proteinprotein interactions. For example, methylation of $\mathrm{G} \gamma \mathrm{T} 1$ is thought to enhance the interaction of the transducin complex with metarhodopsin II (Ohguro et al., 1991). Furthermore, the interaction between C-terminal isoprenylated PDE6 peptides and prenyl binding protein $\delta(\operatorname{PrBP} \delta)$ is influenced by the methylation status of PDE6 (Cook et al., 2000). Methylation has been proposed to be a dynamic modification, but conclusive evidence for reversibility of this modification is still lacking. Early studies suggest that methylation of $\mathrm{G} \gamma \mathrm{T} 1$ is reversible, but experiments purifying transducin from photoreceptors failed to identify a nonmethylated isoform (Fukada et al., 1994; Pérez-Sala et al., 1991).

A requirement for $C A A X$ protein processing in retinal function was demonstrated by a study in which Rcel was inactivated in the neural retina (Christiansen et al., 2011). An absence of RCE1-mediated protein processing disrupted the transport of PDE6 to the OS. That study did not determine whether the defective PDE6 transport in retinal neurons was due to lack of RCE1-mediated processing, the absence of the subsequent methylation step, or the absence of both processing steps.

The importance of ICMT-mediated methyl esterification has been demonstrated with gene-targeted mice where it was observed that inactivation of Icmt caused death during embryonic development (Kim et al., 1999; Bergo et al., 2001). Therefore, to specifically investigate the role of methyl esterification on retinal proteins, we eliminated Icmt expression in the retina with CreloxP recombination techniques. The retina-specific Icmt knockout mice were created by breeding $I c m t^{\mathrm{fl} / \mathrm{fl}}$ mice with mice expressing Cre recombinase under the control of the retina- and forebrain-specific promoter Six3 (Furuta et al., 2000; Bergo et al., 2004). In this study, we combined histology, electroretinography (ERG), and biochemistry to assess the functional relevance of ICMT-mediated protein processing on the function of the photoreceptor neurons.

\section{Materials and Methods}

Mouse models. Mice homozygous for conditional Icmt knock-out alleles $\left(I c m t^{\mathrm{fl} / f \mathrm{l}}\right)$ were bred with mice hemizygous for Six3-Cre transgene to create Icm $t^{\mathrm{fl} / \mathrm{fl}}$ Six 3-Cre mice, which have a retina-specific inactivation of Icmt (Furuta et al., 2000; Bergo et al., 2004). Littermate Icmt ${ }^{\text {wt/fl }}$ Six3-Cre 
A

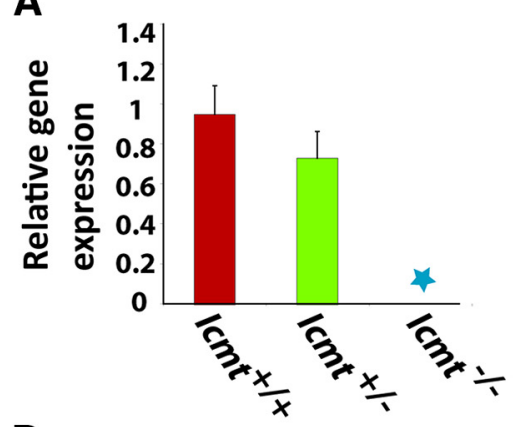

D
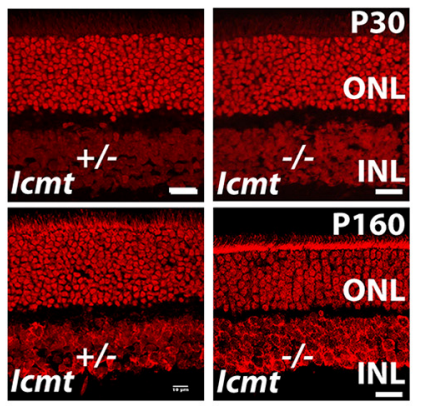

B
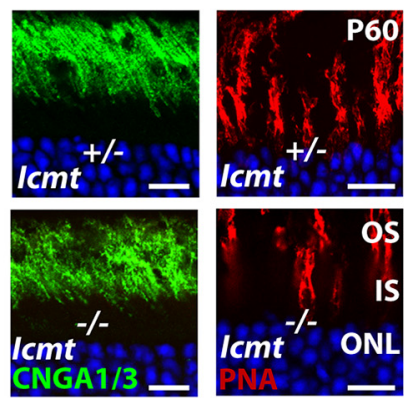

C
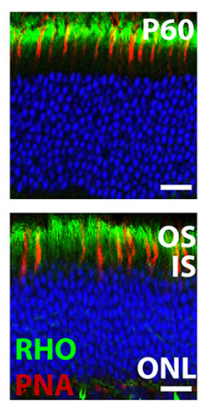

\section{E}

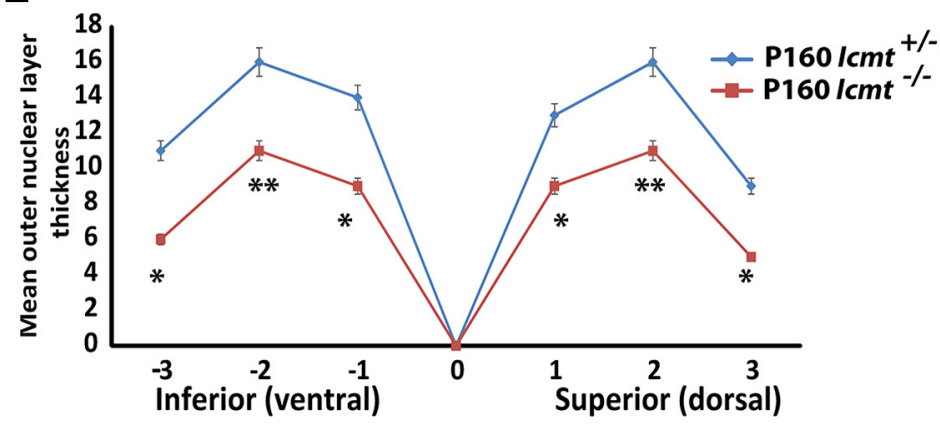

Figure 2. Photoreceptor development is unaltered in the absence of $/ \mathrm{cm}$ expression. A, RT-PCR on P60 retinal CDNA showing the expression of $/ \mathrm{cmt}$ (normalized to hypoxanthine phosphoribosyltransferase, $\mathrm{Hprt})(n=3$, Student's t test, $p=0.033) . \mathrm{lcm}$ primers were designed with the forward primer in exon 1 and reverse primer in exon $4 . B_{,}$(ryosections of P60 /cmt ${ }^{+/-}$and $/ \mathrm{cmt}{ }^{-1-}$ littermate mice labeled with cyclic-nucleotide gated channel $\alpha 1$ (CNGA1/3, green), PNA (red), and T0-PRO-3 (blue). IS, Inner segment; ONL, outer nuclear layer. Scale bar, $5 \mu \mathrm{m}$. C, Cryosections of P60 $\mathrm{cmt}^{+/-}$and $/ \mathrm{cmt}^{-/-}$littermates labeled with RHO (green), PNA (red), and T0-PR0-3 (blue). Scale bar, $10 \mu \mathrm{m}$. D, Cryosections of P30 (top) and P160 (bottom) $/ \mathrm{cmt}{ }^{+/-}$and $/ \mathrm{cmt}{ }^{-/-}$ littermate mice labeled with propidium iodide, a nuclear stain. $E$, Spyder plot analysis of ONL thickness at P160 $(n=4) .{ }^{*} p<0.004$ (Student's $t$ test). ${ }^{* *} p<0.002$ (Student's $t$ test).

mice were used as controls. Both males and females were used in the study. Genotyping was performed by PCR amplification using genomic DNA extracted from mouse ear punch biopsies as described previously (Court et al., 2013). Mouse experiments were performed in accordance with the National Institutes of Health guidelines and the protocol approved by Institutional Animal Care and Use Committee of West Virginia University.

Quantitative PCR. Retinas were dissected from freshly enucleated eyes and frozen on dry ice in the presence of Trizol (Invitrogen). RNA extracted from frozen retinas was used to generate cDNA with Qscript (Quanta Bioscience). A total of $300 \mu \mathrm{g}$ of cDNA from heterozygous and knock-out littermates (in triplicate) was used as a template for quantitative PCR using MyiQ PCR cycler (Bio-Rad) and MyiQ SYBR Green Supermix (Bio-Rad). Icmt was amplified with primers $5^{\prime}$-CGCCTCAGCCTCGCTACATT-3' (exon 1) and 5'-TTGGAGCCAGCCGTAAACAT-3' (exon 4), yielding a 509 bp product. Threshold values were normalized to hypoxanthine phosphoribosyltransferase (Hprt) gene expression levels with primers 5'CAAACTTTGCTTTCCCTGGT-3' and 5'-CAAGGGCATATCCAACA ACA-3' (250 bp product).

ERG. Littermates were dark-adapted overnight, and the eyes were dilated (1:1 phenylephrine/tropicamide) for $10 \mathrm{~min}$. Isoflurane anesthesia ( $1.5 \%$ in $2.5 \%$ oxygen) was administered via nose cone on a $37^{\circ} \mathrm{C}$ platform. A reference electrode was placed subcutaneously in the scalp, and silver wire electrodes were positioned above the cornea, with contact being made by methylcellulose solution. Light flashes were presented by placing the mouse in a Ganzfield apparatus. Corneal-evoked potentials were collected using UTAS-E4000 Visual Electrodiagnostic Test System and EMWIN 8.1.1 software (LKC Technologies). Background light $\left(30 \mathrm{~cd} \cdot \mathrm{m}^{-2}\right)$ was presented for $10 \mathrm{~min}$ before recording flicker responses in the presence of the background light. Representative waveforms are shown.

Immunoblotting. Flash frozen retinal samples were solubilized in $1 \times$ PBS-containing protease inhibitor (Pierce protease inhibitor, Thermo Scientific) using sonication for 4 pulses of $20 \mathrm{~ms}$ at power setting 6 (Misonix XL-2000). The protein concentration was estimated with a NanoDrop (Thermo Scientific) spectrophotometer. Protein samples
$(150 \mu \mathrm{g})$ were size-fractionated on $4 \%-20 \%$ Criterion (Bio-Rad) polyacrylamide gels. Proteins were then transferred to Immobilon-FL membrane (Millipore) and incubated with primary antibodies against specific proteins. The following antibodies were used: anti-PDE6 $\alpha$ (Cytosignal), anti-PDE6 $\beta$ (Thermo Fisher), anti-G $\gamma \mathrm{T} 1$ (Santa Cruz Biotechnology), anti-G $\gamma \mathrm{T} 2$ (gift from Dr. Vadim Arshavsky, Duke University Eye Center, Durham, NC), anti-GRK1 (Thermo Fisher), anti-G $\alpha$ T1and anti-G $\alpha$ T2 (Santa Cruz Biotechnology), anti-G $\beta \mathrm{T} 1$ (Santa Cruz Biotechnology), anti-arrestin (Affinity Bioreagents), and anti-PDE6 $\alpha^{\prime}$ (Thermo Fisher). To detect primary antibodies, we used Odyssey goat anti-rabbit Alexa680 and Odyssey goat anti-mouse Alexa-680 secondary antibodies (LICOR Biosciences) at 1:50,000 dilutions. Membranes were scanned with an Odyssey Infrared Imaging System (LI-COR Biosciences). Images are representative from three or more independent experiments.

Immunohistochemistry. Mice were killed by $\mathrm{CO}_{2}$ inhalation, and eyes were enucleated. A $2 \mathrm{~mm}$ hole was made at the corneal limbus, and eyes were fixed with 4\% PFA for 10 min before dissecting the anterior chamber and removing the lens. Eyecups for cryosections were then fixed for $50 \mathrm{~min}$ in 4\% PFA in PBS solution before cryoprotection in $20 \%$ sucrose overnight at $4^{\circ} \mathrm{C}$. Eyecups were embedded in Tissue-Tek optimal cutting temperature compound (Sakura) and fast-frozen in dry ice ethanol bath. Blocks were sectioned with a Leica CM1850 Cryostat, and $18 \mu \mathrm{m}$ sections were mounted on Superfrost plus slides. Cryosections were washed in PBS solution and then incubated in blocking buffer [ $2 \%$ goat serum (Invitrogen), $0.1 \%$ Triton $\mathrm{X}-100$, and $0.05 \%$ sodium azide in PBS solution] for $1 \mathrm{~h}$. Primary antibodies were incubated for $4 \mathrm{~h}$ at room temperature or overnight at $4^{\circ} \mathrm{C}$. Excess antibody was removed by three 10 min washes in PBS solution with $0.1 \%$ Triton $\mathrm{X}-100$ before incubation with secondary antibody for $45 \mathrm{~min}$ at room temperature. Slides were washed twice for 10 min with PBS solution with $0.1 \%$ Triton X-100 and for $10 \mathrm{~min}$ in PBS solution. ProLong Gold antifade reagent (Invitrogen) was applied to each section, and then coverslips were mounted. Images were collected on a Zeiss LSM 510 Meta confocal microscope using 488,543 , and $633 \mathrm{~nm}$ laser lines. The following antibodies were used: anti-cyclic nucleotide-gated channel (CNGA1/3) (University of Cal- 
A

P24

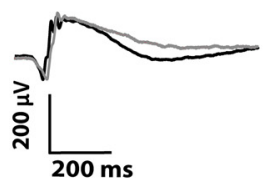

$200 \mathrm{~ms}$

B

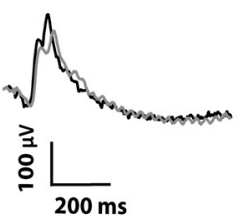

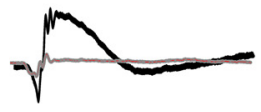

P160

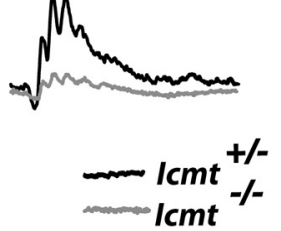

C

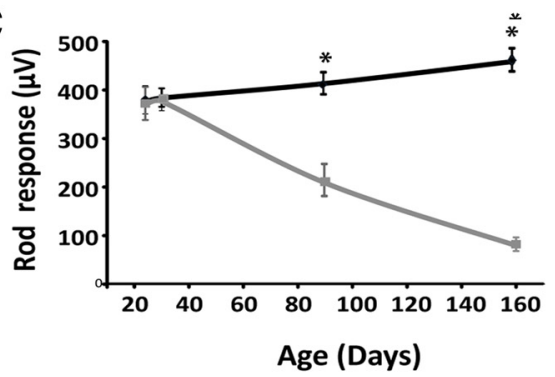

D

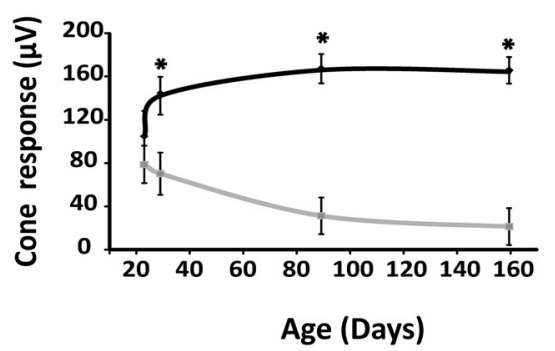

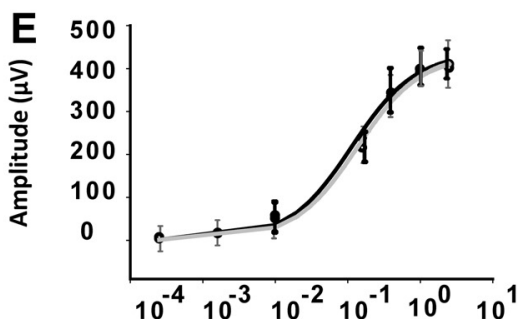

Flash Strength $\left(\mathrm{cd}^{*} \mathrm{~s} / \mathrm{m}^{2}\right)$

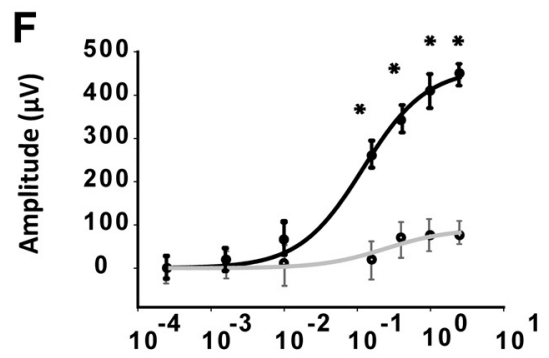

Flash Strength $\left(\mathrm{cd}^{*} \mathrm{~s} / \mathrm{m}^{2}\right)$

Figure 3. Visual deficit in mice lacking $/ \mathrm{cm} t$ in the retina. $\operatorname{Rod}(\boldsymbol{A})$ and cone $(\boldsymbol{B})$ ERG responses recorded from P24 and P160 $/ \mathrm{cmt} t^{+/-}$and $/ \mathrm{cmt} t^{-/-}$littermates $(\mathrm{n}=4)$. $\boldsymbol{C}, \boldsymbol{D}, A m p l i t u d e$ of "a" and " $b$ " waves versus age (in days). Rod responses $(\boldsymbol{C}$ correspond to the amplitude of the " $a$ " wave, whereas cone responses (D) reflect the amplitude of the "b" wave. Representative rod waveforms measured at $-0.8 \log \mathrm{cd}^{*} \mathrm{~s} / \mathrm{m}^{\wedge} 2$; representative cone waveforms measured at $0.7 \log \mathrm{cd}^{*} \mathrm{~s} / \mathrm{m}^{\wedge} 2(n=4$, Student's $t$ test, $p<0.002)$. P, Postnatal days; $n$, number of littermates. $\boldsymbol{E}, \boldsymbol{F}$, Intensity response relations of scotopic "a" waves at P24 and P160 lcmt ${ }^{+/-}$and $/ \mathrm{cmt} t^{-1-}$ littermates $(n=4)$. The data were fitted with the hyperbolic functions that yielded scotopic "a" wave half-saturating light intensities of $0.10 \pm 0.02 \mathrm{~cd}^{*} \mathrm{sm}^{-2}$ for $\mathrm{lcmt} \mathrm{t}^{+-}$and $0.11 \pm 0.034 \mathrm{~cd}^{*} \mathrm{sm}^{-2}$ for Icmt ${ }^{-/-}$littermates, respectively, at P24 (E). The "a" wave maximum amplitudes were $413.22 \pm 14.32 \mu \mathrm{V}\left(\mathrm{lcmt}{ }^{+/-} ; n=4\right)$ and $398.79 \pm 8.21 \mu \mathrm{V}\left(\mathrm{Icmt}{ }^{-1-} ; n=4\right)$. Similarly at P160, data were fitted with the hyperbolic functions that yielded scotopic "a" wave half-saturating light intensities of $0.11 \pm 0.02 \mathrm{~cd}^{*} \mathrm{sm}^{-2}$ for $/ \mathrm{cmt}^{+/-}$and $0.24 \pm 0.12 \mathrm{~cd}^{*} \mathrm{sm}^{-2}$ for $\mathrm{lcmt}{ }^{-1-}$ littermates, respectively, at P160 $(\boldsymbol{F})$. The "a" wave maximum amplitudes were $461.17 \pm 16.11 \mu \mathrm{V}$ $\left(\mathrm{lcmt} t^{+/-} ; n=4\right)$ and $90.12 \pm 13.21 \mu \mathrm{V}\left(\mathrm{lcmt}{ }^{-/-} ; n=4\right)$. Data are mean \pm SEM. ${ }^{*} p<0.004$ (Student's $t$ test)

A

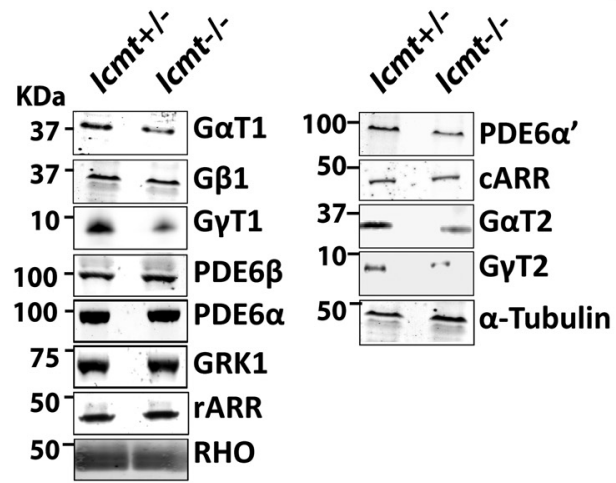

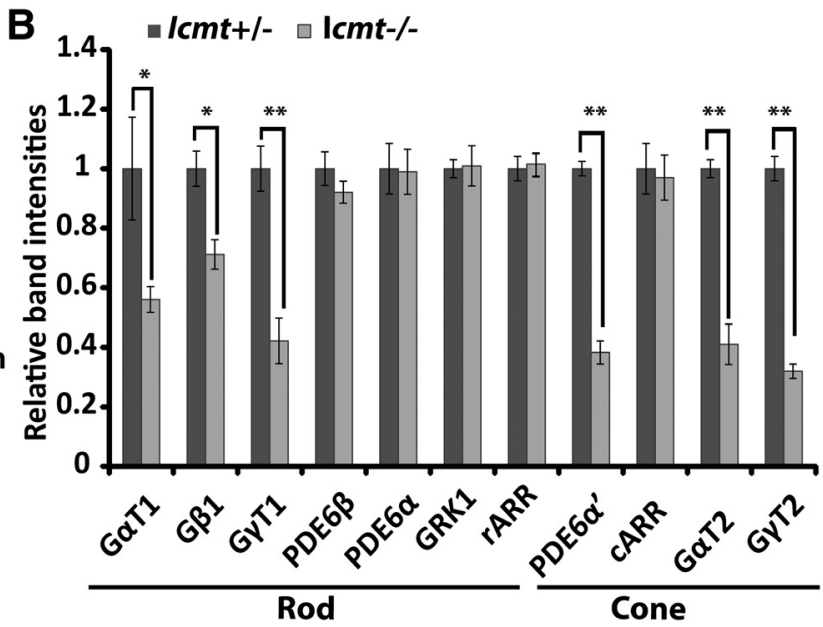

Figure 4. Levels of a subset of isoprenylated photoreceptor proteins are reduced in the absence of ICMT-mediated protein processing. Immunoblots using P30 retinal lysates. $A$, Western blots of protein extracts probed with antibodies against the indicated proteins. B, Quantitative comparison of indicated photoreceptor proteins between $/ \mathrm{cmt}$-deficient mice and littermate controls. $y$ axis represents the percentage of the integrated intensity measurement normalized to measurements in $1 \mathrm{cmt}^{+/-}$mice (as judged by Odyssey imaging software). Integrated intensity values were normalized to $\alpha$-tubulin $(n=4) .{ }^{*} p<0.004$ (Student's $t$ test). ${ }^{* *} p<0.002$ (Student's $t$ test).

ifornia-Davis/National Institutes of Health NeuroMab Facility), anti-PDE $\beta$ (Affinity Bio Reagents), anti-G $\gamma \mathrm{T} 1$ (Santa Cruz Biotechnology), anti-G $\gamma$ T2 (gift from Dr. Vadim Arshavsky, Duke University Eye Center, Durham, NC), anti-PDE6 $\alpha^{\prime}$ (Thermo Fisher), anti-GRK1 (Ching-Kang Chen, Virginia Commonwealth University, Richmond, VA), and TO-PRO-3 nuclear stain (Invitrogen), added to dilutions of AlexaFluor secondary antibodies (Invitrogen) in antibody dilution buffer $(0.05 \%$ goat serum, $0.1 \%$ Triton X-100, and $0.05 \%$ sodium azide in $1 \times$ PBS solution.

Membrane fractionation. Cell fractionation was performed as described previously (Kolandaivelu et al., 2014). Briefly, flash-frozen retinal samples were homogenized in $1 \times$ PBS-containing protease inhibitors (Pierce protease inhibitor, Thermo Scientific) by sonication with 5 pulses of $15 \mathrm{~ms}$ at power setting 6 (Misonix XL-2000). After centrifugation at $5000 \times g$ for $5 \mathrm{~min}$ at $4^{\circ} \mathrm{C}$, the supernatant (total fraction) was collected and cellular debris was discarded. The low-speed supernatant was then spun at $45,000 \times g$ for 30 min (Rotor TLA-55 Beckman Coulter) to isolate the soluble fraction. After removal of the high-speed supernatant (cytosol-soluble fraction), the pellet was resuspended in an equal volume of $1 \times$ PBS (membrane fraction). All protein samples were then analyzed by SDS-PAGE followed by immunoblotting to check for the distribution of cytosolic and membrane proteins. 
A

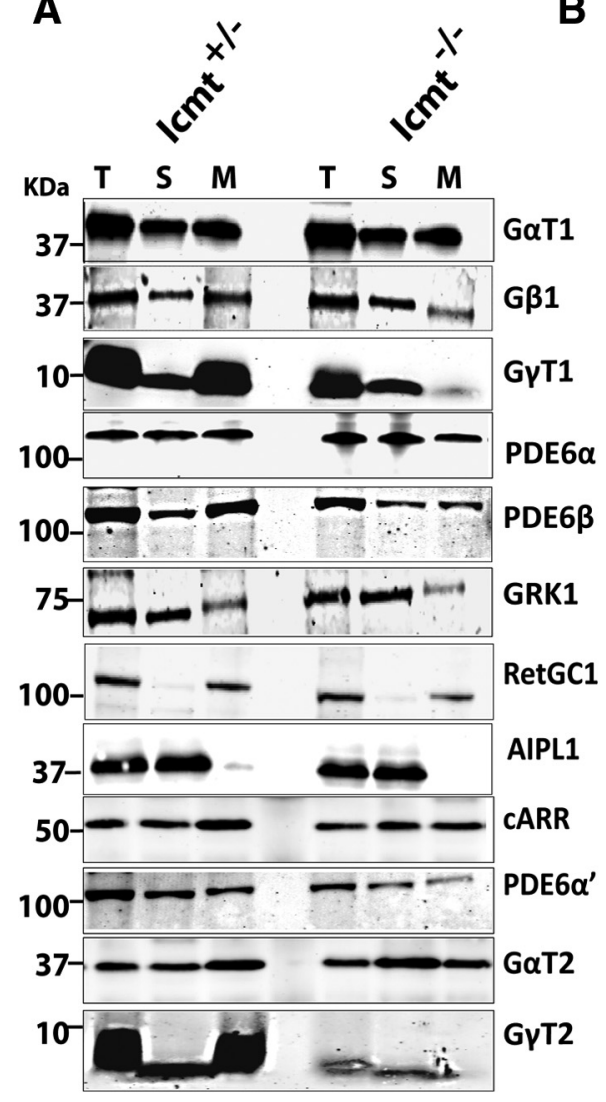

B
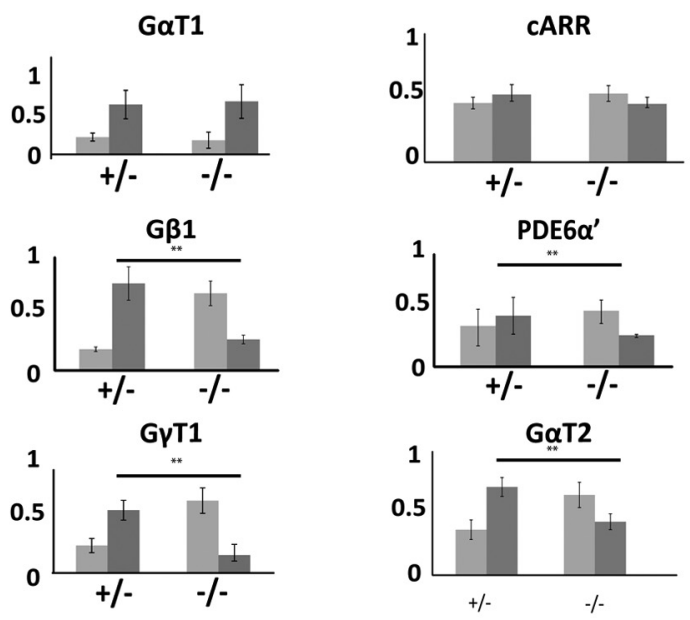

PDE6 $\beta$
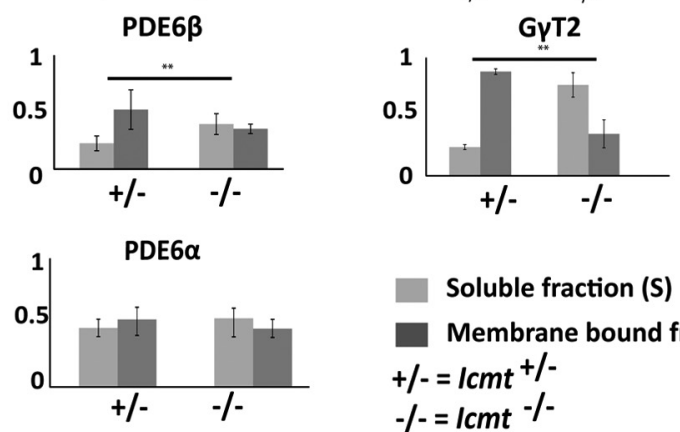

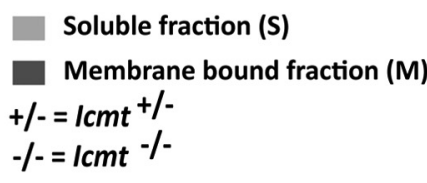

Figure 5. Membrane association of transducin and cone PDE6 is impaired in retinae lacking / cmt expression. A, Isotonic membrane fractionation of P30 retinal lysates, followed by Western blotting. AIPL1, RetGC1, and Cone Arrestin (CARR) serve as controls $(n=4)$. B, Quantitative comparison of indicated proteins in membrane-bound and soluble fractions in $/ \mathrm{cm}$ knock-out mice and littermate controls. $y$-axis represents the percentage of the integrated intensity measurements normalized to measurements in control ( $\left(\mathrm{cmt}^{+/-}\right)$samples, as judged with 0 dyssey imaging software. Integrated intensity values were normalized to $\alpha$-tubulin $(n=4) .{ }^{* *} p<0.05$. T, Total fraction; S, soluble fraction; M, membrane-bound fraction.

\section{Results}

Generating mice lacking Icmt expression in the retina

To study the role of methyl esterification of proteins in the retina, we generated retina-specific Icmt knock-out mice. Six3-Cre expression eliminates Icmt expression in the retina and the forebrain at embryonic day 9.5 (Furuta et al., 2000; Christiansen et al., 2011). Icmt $t^{\mathrm{f} / \mathrm{wt}}$ Six3-Cre males (hereafter referred to as Icmt ${ }^{+/-}$ mice) were mated with $I c m t^{\mathrm{f} / \mathrm{fl}}$ females to generate $I c m t^{\mathrm{fl} / \mathrm{fl}} \operatorname{Six} 3$ Cre mice (hereafter referred to as Icmt ${ }^{-1-}$ mice, as well as littermate controls $\left[I c m t^{\mathrm{t} / / \mathrm{wt}}\right.$ Six3-Cre $\left(I c m t^{+/-}\right) ; \operatorname{Icm} t^{\mathrm{f} / \mathrm{fl}}, I \mathrm{Icm} t^{\mathrm{wt} / \mathrm{ll}}$ $\left.\left(\mathrm{Icmt} \mathrm{C}^{+/+}\right)\right]$. Quantitative RT-PCR was performed to confirm the absence of Icmt transcripts in the retina of Icmt ${ }^{-1-}$ mice (Fig. $2 \mathrm{~A}$ ). For these studies, $\mathrm{Icmt}{ }^{+/-}$or $\mathrm{Icmt}{ }^{+/+}$littermates were used as controls; those mice were similar to C57BL/6J animals with identical photoreceptor responses (data not shown).

\section{ICMT-mediated protein processing is not required for} photoreceptor morphogenesis

To test the role of ICMT-mediated protein processing on photoreceptor development, we assessed the presence of rods and cones with cell-specific markers (Fig. 2B). Rod and cone photoreceptor cells were present in a normal distribution in retinas of $I \mathrm{~cm} t^{-1-}$ mice as shown by the presence of CNGA1/3 (a marker for rod and cone cells) and peanut agglutinin (PNA, a marker for cone cells) (Fig. 2B, top, bottom). Photoreceptor morphology appeared normal by electron microscopy (data not shown). In addition, rod opsin (RHO) labeling demonstrated normal development of rod OSs (Fig. 2C). We did not observe any changes in the thick- ness of the outer nuclear layer at P30 (Fig. 2D, top). However, photoreceptors exhibited a slow and progressive loss of three or four nuclear layers (Student's $t$ test, $p \leq 0.005$ ) by P160 (Fig. 2D, bottom $E$ ). Together, our data show that methylation of isoprenylated proteins is not required for photoreceptor development.

Light-evoked response is progressively reduced in retinas of Icmt-deficient mice

ERG was used to measure the response of photoreceptor cells in $\mathrm{Icmt} \mathrm{C}^{+/-}$and $\mathrm{Icmt}{ }^{-1-}$ mice to flashes of increasing light intensities. The $a$-wave of scotopic ERGs is generated by hyperpolarization of rod photoreceptor cells in response to light. Subsequent signaling to downstream neurons leads to depolarization of postsynaptic bipolar cells, which is measured by the $b$-wave of ERGs. At P24, there were no significant changes in rod or cone responses in mice lacking Icmt. This finding is in agreement with our observation that photoreceptor development is normal in Icmt ${ }^{-1-}$ mice. However, as the mice age, rod responses progressively declined. At P160, maximal rod responses were reduced by $82 \%$ $(n=4$, Student's $t$ test, $p=0.032)$ and cone responses were reduced by $92 \%$ (Fig. 3$)(n=4$, Student's $t$ test, $p=0.017)$. We also plotted the loss of visual response at various ages, which illustrated progressive loss of photoreceptor function over time (Fig. 3C,D). In comparison with rods, cones exhibit greater functional loss and were more sensitive to ICMT deficiency (Fig. $3 C, D)$. We also checked the sensitivity of ERG response at different light intensities (Fig. 3E,F). Strikingly, we did not observe any significant changes in light sensitivity at P24. At P160, the 
sensitivity of ERG response showed a slight reduction in mice lacking ICMT. Collectively, these results show that rod and cone signal transduction pathways are disrupted in aged $\mathrm{Icmt}^{-1-}$ mice.

Levels of a subset of isoprenylated photoreceptor proteins were reduced in the absence of ICMT-mediated protein processing

We reasoned that a reduced visual response might result from lower levels of the isoprenylated proteins involved in phototransduction. Therefore, we assessed protein levels by Western blotting with retinal extracts from $I \mathrm{~cm} t^{+/-}$and Icmt ${ }^{-1-}$ mice at P30 (before significant retinal degeneration was evident). We observed more than a $60 \%$ reduction $(n=4$, Student's $t$ test, $p=0.0011$ ) in levels of the cone PDE6 (PDE6 $\left.\alpha^{\prime}\right)$ and rod and cone transducin- $\gamma$ subunits ( $\mathrm{G} \gamma \mathrm{T} 1$ and $\mathrm{G} \gamma \mathrm{T} 2$, respectively) in $\mathrm{Icmt}^{-1-}$ mice (Fig. $4 \mathrm{~A}, \mathrm{~B}$ ). In contrast to our study of retina-specific Rce 1 knock-out mice, we did not observe any changes in the electrophoretic mobility of isoprenylated proteins (Christiansen et al., 2011). Surprisingly, the levels of isoprenylated rod PDE6 catalytic subunits $(\mathrm{PDE} 6 \alpha \beta)$ and GRK1 were unaffected. As expected, rod arrestin, a photoreceptor protein that is not isoprenylated, was unaffected (Fig. 4A,B). The transducin $\alpha(\mathrm{G} \alpha \mathrm{T} 1)$ subunits, which form a heterotrimeric complex with $\mathrm{G} \beta 1-\mathrm{G} \gamma \mathrm{T} 1$, were reduced in the absence of ICMT-mediated protein methylation. We also assessed protein levels at P16 (well after Six3-Cremediated recombination, which occurs at E9.5) and did not observe significant changes (data not shown). Our results suggest that lack of cysteine methylation by ICMT causes photoreceptor $C A A X$ proteins to undergo increased protein turnover, with transducin subunits and PDE6 $\alpha^{\prime}$ showing the most pronounced reductions in their steady-state levels.

\section{Membrane association of transducin and cone PDE6 is} affected in retinas lacking Icmt expression

We hypothesized that impaired association of unmethylated G $\gamma \mathrm{T} 1 / 2$ and PDE6 $\alpha^{\prime}$ with membranes might contribute to their lower levels in $\mathrm{Icmt}^{-1-}$ mice. To examine the effect of ICMTmediated methylation on membrane attachment of proteins, we performed isotonic membrane fractionation of retinal extracts. Our results show that the majority (77\%) of G $\gamma \mathrm{T} 1$ in control $\left(\mathrm{Icmt} \mathrm{C}^{+-}\right)$mice was present in the membrane fraction. In contrast, $<10 \%$ of G $\gamma \mathrm{T} 1$ was in the membrane fraction in $\mathrm{Icmt}^{-1-}$ mice ( $n=4$, Student's $t$ test, $p=0.042)$. The membrane attachment of $\mathrm{G} \beta \mathrm{T} 1$, a partner of $\mathrm{G} \gamma \mathrm{T} 1$, was similarly affected (Fig. $5 A, B)$. A similar reduction in membrane association of cone transducin subunits (G $\gamma \mathrm{T} 2$ and $\mathrm{G} \alpha \mathrm{T} 2)$ was also detected. Defective membrane association of cone PDE6 $\alpha^{\prime}$ and geranylgeranylated $\operatorname{rod}$ PDE6 $\beta$ in $\mathrm{Icmt}^{-1-}$ animals was also observed (Fig. $5 A, B)$. Surprisingly, the membrane association of GRK1 and PDE6 $\alpha$, which are farnesylated proteins, was not affected by the absence of methylation. Guanylate cyclase-1 (RetGC1) and aryl hydrocarbon receptor interacting protein like-1 (AIPL1) were used as membrane-bound and cytosolic protein controls, respec- tively, and were unaffected by the deficiency in ICMT-mediated protein processing (Fig. 5). Overall, our results demonstrate the importance of methylation in altering the membrane binding of the visual G-protein, transducin, and PDE6.

\section{Trafficking of isoprenylated OS proteins are not affected by} the absence of ICMT-mediated methylation

Our earlier study revealed the importance of RCE1-mediated endoproteolysis in retinal neurons (the processing step that precedes the ICMT-mediated methylation step). We found that the PDE6 holoenzyme is assembled but does not traffic to the OS in the Rce $1^{-1-}$ mice (Christiansen et al., 2011). The defective transport of PDE6 could be due to lack of endoproteolysis, the absence of the subsequent methylation step, or the absence of both steps. To distinguish between these possibilities, we examined the trafficking of PDE6 and other isoprenylated OS proteins in Icmtdeficient mice. Immunolocalization of PDE6 $\beta$ was compared with that of CNGA1/3, a marker of OS (Fig. $6 A, B$ ). Merged images demonstrated that PDE6 was transported to the OS and colocalized with CNGA1/3 in retinas lacking Icmt expression. Similarly, G $\gamma$ T1 was localized in the OS with CNGA1/3 (Fig. 6B). In addition, no significant defects in localization of cone-specific PDE6 $\alpha^{\prime}, \mathrm{G} \gamma \mathrm{T} 2$, and GRK1 (present in both rods and cones) were observed at P30 (Fig. 6C,D).

Despite marked reduction in photopic ERG response at $\mathrm{P} 160$, cone density in Icmt-deficient retina was unaltered. Interestingly, GRK1 was severely reduced in cones, whereas expression of GRK1 in rods did not show any significant changes (Fig. 7B). A drastic reduction in cone PDE6 and GyT2 levels was also observed at $\mathrm{P} 160$ in Icmt-deficient mice (Fig. $7 \mathrm{~A}, \mathrm{~B}$ ). The results from immunolocalization studies were supported by our immunoblotting analysis (Fig. 7C) and demonstrate the importance of methylation for the stability of isoprenylated proteins. Our re- 

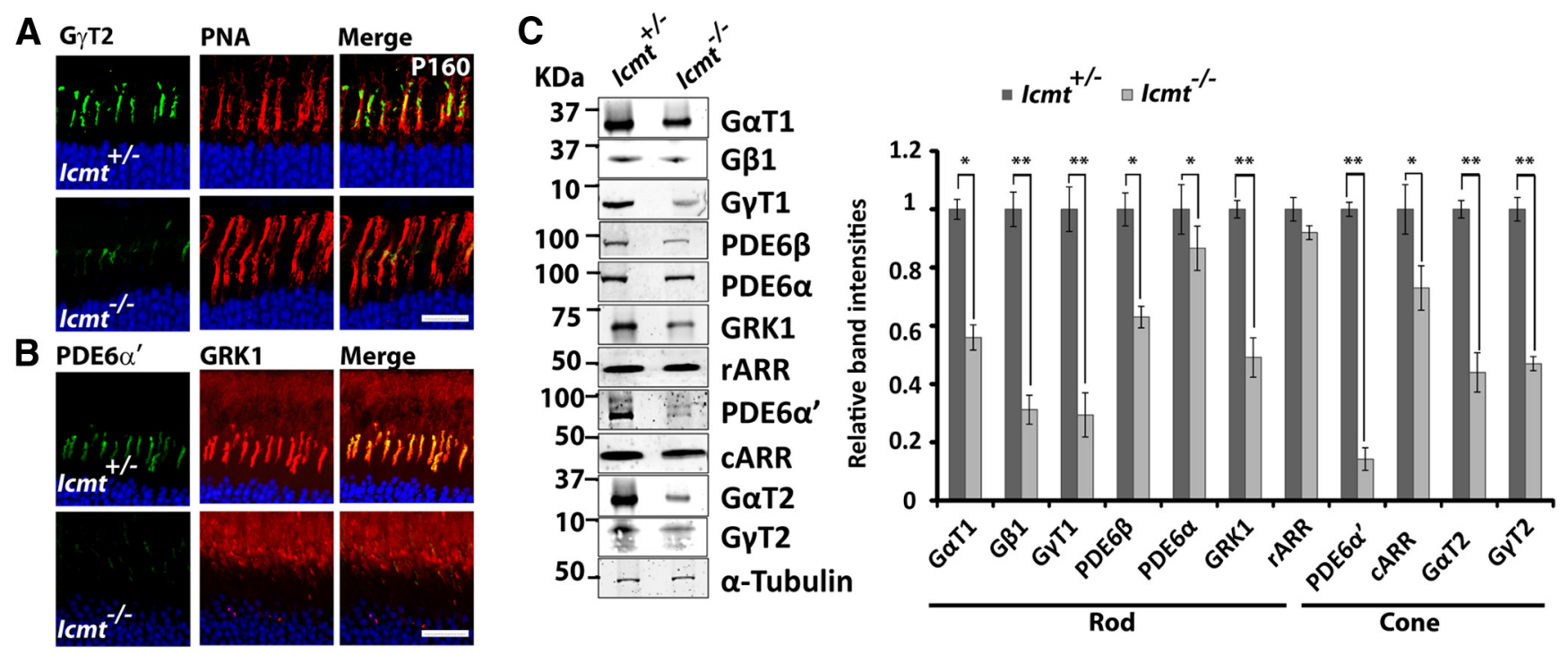

Figure 7. PDE6 and GRK1 levels in cones are reduced in older mice lacking ICMT-mediated protein methylation. Immunofluorescent labeling of P160 /cmt ${ }^{+/-}$and $/ \mathrm{cm}^{-/-}$littermates. $\boldsymbol{A}_{\text {, Red }}$ represents PNA in the cone sheath. Green represents $G \gamma T 2$, colocalizes with PNA. Blue represents nuclei, stained with T0-PR0-3. B, Red represents GRK1. Green represents cone PDE6 (PDE6 $\alpha^{\prime}$ ). Both GRK1 and cone PDE6 are absent in Icmt ${ }^{-1-}$ at P160. Scale bar, $10 \mu \mathrm{m}$. C, Representative Western blots of protein extracts probed with antibodies against the indicated proteins (left) and quantitative comparison of indicated proteins between $/ \mathrm{cmt}^{-1-}$ mice and littermate controls (right). $y$-axis represents the percentage of the integrated intensity measurements normalized to measurements in control $\left(/ \mathrm{cmt}^{+/-}\right)$samples, as judged with Odyssey imaging software $(n=4)$. Integrated intensity values are normalized to $\alpha$-tubulin $(n=4)$. ${ }^{*} p<0.05$ (Student's $t$ test). ${ }^{* *} p<0.02$ (Student's $t$ test).

sults show that methylation of proteins has little or no effect on the ability of isoprenylated proteins to traffic to the OS, but it is crucial for the stability of isoprenylated OS proteins.

\section{Discussion}

Our current studies highlight the importance of post-translational modifications of proteins in the function and survival of photoreceptor neurons. The most significant defect in mice lacking ICMT-mediated protein methylation in the retina is the loss of visual function accompanied by slow photoreceptor degeneration.

Methylation of proteins by Icmt was not required for retinal development, despite early inactivation of Icmt in the retina. The characteristic layering of neurons in the retina was preserved, and all retinal layers were formed normally. In addition, we found no alteration in the ultrastructure of rod and cone photoreceptors by electron microscopy (data not shown). These findings are in agreement with normal photoreceptor ERG response at P24. However, we observed progressive reduction in rod and cone responses with extinguished photoreceptor responses by 5 months of age. There appeared to be discordance between the striking extent of functional deficit and the loss of only 3 or 4 layers of photoreceptor nuclei at 5 months. We attribute the defective rod response to loss of transducin, although the cone responses were affected by the loss of both transducin and phosphodiesterase, two crucial players in phototransduction. This interpretation is supported by studies where elimination of transducin ( $\mathrm{G} \gamma \mathrm{T} 1)$ expression resulted in late-onset retinal degeneration and reduced rod ERG responses (Lobanova et al., 2008; Kolesnikov et al., 2011). A mouse model defective in cone PDE6 showed similar reduction in photopic ERG (Chang et al., 2006; Kolandaivelu et al., 2011). Interestingly, the survival of the neurons in the inner retinal layer was unaffected by the absence of Icmt expression.

The effect of ICMT-mediated protein methylation on the stability of isoprenylated proteins is variable (Bergo et al., 2000). For instance, RhoA undergoes higher rates of turnover in the absence of methylation, whereas K-Ras is more stable in the absence of methylation (Bergo et al., 2004). The levels of cone PDE6 $\alpha^{\prime}$ in Icmt ${ }^{-1-}$ mice were reduced significantly (60\%-70\%), in contrast to normal expression of rod PDE6 before degeneration at P30 (Fig. 4). Interestingly, as the photoreceptors degenerate by four nuclear layers at P160, we observed a major reduction in several phototransduction proteins, such as rod and cone PDE6, cone GRK1, and transducin (Fig. 7). Along with PDE6, our immunolocalization studies showed markedly reduced levels of GRK1 in cones (Fig. 7). The reason for the selective reduction in cone PDE6 subunits before degeneration is not clear. Cone PDE6 subunits are geranylgeranylated, whereas the obligatory catalytic heteromer of rod PDE6 is farnesylated $(\alpha)$ or geranylgeranylated $(\beta)$ (Anant et al., 1992). Similarly, the reason for the selective reduction in farnesylated GRK1 in cones at P160 is not clear. It is known that methylation of PDE6 is crucial for its interaction with $\operatorname{PrBP} \delta$, a prenyl-binding protein, and is needed for stability and trafficking of PDE6 and GRK1 in photoreceptors (Zhang et al., 2007, 2012; Cook et al., 2000). Overall, our data support a model where methylation of cone PDE6 and GRK1 is essential for the interaction of these proteins with $\operatorname{PrBP} \delta$, and the absence of this interaction in Icmt-deficient cones leads to reduced cone photoreceptor function.

The results from this study also show a requirement for protein methylation in maintaining normal levels of the G-protein transducin complex $(\alpha \beta \gamma)$. Unmethylated transducin $(\mathrm{G} \beta \gamma \mathrm{T} 1)$ is defective in its association with retinal membranes (Fig. 4). This finding is in agreement with a previous in vitro study that demonstrated the importance of protein methylation in anchoring of G $\beta \gamma \mathrm{T} 1$ to rod OS membranes (Fukada et al., 1990; Ohguro et al., 1991). However, the membrane fractionation pattern of $\mathrm{G} \alpha \mathrm{T} 1$, a myristoylated protein, was unaltered, suggesting that the assembly of the G-protein transducin complex is affected in the absence of methylation. The reduced rod response is likely a re- 
sult of deficiencies in both transducin complex formation and inadequate membrane association of $\mathrm{G} \beta \gamma \mathrm{T} 1$. The puzzling finding in our studies was the normal photo-responses at P24 despite defective G $\gamma \mathrm{T} 1$ membrane association. One explanation for the progressive loss of rod ERGs could be the accumulation of soluble unmethylated $\mathrm{G} \beta \gamma \mathrm{T} 1$ in the rod OS over time that interferes with $\mathrm{RHO}^{\star}$-mediated light signaling.

Phototransduction proteins are synthesized, assembled in the inner segment, and then transported to OS. However, methylation of isoprenylated proteins does not affect the ability of phototransduction proteins to traffic to the OS. This observation stands in contrast to our findings with $\mathrm{Rce}^{-/-}$mice, where rod and cone PDE6 accumulated in the inner segment. Based on these results, we conclude that the retention of the $-A A X$ in PDE6 catalytic subunits in the setting of RCE1 deficiency impairs PDE6 transport to the OS. Our findings showing severe retinal disease phenotypes in $R c e 1^{-1-}$ mice also stand in contrast to published reports on global Icmt and Rcel knock-out mice. A global deficiency of Icmt results in embryonic death much earlier in development than a deficiency of Rcel (Kim et al., 1999; Bergo et al., 2001, 2004; Michaelson et al., 2005). Although it seems unlikely, we cannot rule out the possibility that a distinct methyltransferase might retain the capacity to methylate certain proteins after inactivation of Icmt in the retina. If there were another methyltransferase, it could potentially help to explain the fact that retinal phenotypes were less severe in mice lacking ICMT than in mice lacking RCE1, despite the fact that ICMT has a larger number of protein substrates (CAAX proteins plus CXC Rab proteins).

Overall, the current investigations revealed that ICMT-mediated protein methylation is essential for stability and membrane anchorage of isoprenylated $\mathrm{G} \beta \gamma \mathrm{T} 1$ and cone PDE6 and is required for photoreceptor function and survival.

\section{References}

Anant JS, Ong OC, Xie HY, Clarke S, O’Brien PJ, Fung BK (1992) In vivo differential prenylation of retinal cyclic GMP phosphodiesterase catalytic subunits. J Biol Chem 267:687-690. Medline

Bergo MO, Leung GK, Ambroziak P, Otto JC, Casey PJ, Young SG (2000) Targeted inactivation of the isoprenylcysteine carboxyl methyltransferase gene causes mislocalization of K-Ras in mammalian cells. J Biol Chem 275:17605-17610. CrossRef Medline

Bergo MO, Leung GK, Ambroziak P, Otto JC, Casey PJ, Gomes AQ, Seabra MC, Young SG (2001) Isoprenylcysteine carboxyl methyltransferase deficiency in mice. J Biol Chem 276:5841-5845. CrossRef Medline

Bergo MO, Gavino BJ, Hong C, Beigneux AP, McMahon M, Casey PJ, Young SG (2004) Inactivation of Icmt inhibits transformation by oncogenic K-Ras and B-Raf. J Clin Invest 113:539-550. CrossRef Medline

Chang B, Dacey MS, Hawes NL, Hitchcock PF, Milam AH, Atmaca-Sonmez P, Nusinowitz S, Heckenlively JR (2006) Cone photoreceptor function loss-3, a novel mouse model of achromatopsia due to a mutation in Gnat2. Invest Ophthalmol Vis Sci 47:5017-5021. CrossRef Medline

Christiansen JR, Kolandaivelu S, Bergo MO, Ramamurthy V (2011) RASconverting enzyme 1-mediated endoproteolysis is required for trafficking of rod phosphodiesterase 6 to photoreceptor outer segments. Proc Natl Acad Sci U S A 108:8862-8866. CrossRef Medline

Cook TA, Ghomashchi F, Gelb MH, Florio SK, Beavo JA (2000) Binding of the $\delta$ subunit to rod phosphodiesterase catalytic subunits requires methylated, prenylated C-termini of the catalytic subunits. Biochemistry 39: 13516-13523. CrossRef Medline

Court H, Amoyel M, Hackman M, Lee KE, Xu R, Miller G, Bar-Sagi D, Bach EA, Bergö MO, Philips MR (2013) Isoprenylcysteine carboxylmethyltransferase deficiency exacerbates KRAS-driven pancreatic neoplasia via Notch suppression. J Clin Invest 123:4681-4694. CrossRef Medline

Fukada Y, Takao T, Ohguro H, Yoshizawa T, Akino T, Shimonishi Y (1990)
Farnesylated gamma-subunit of photoreceptor $\mathrm{G}$ protein indispensable for GTP-binding. Nature 346:658-660. CrossRef Medline

Fukada Y, Matsuda T, Kokame K, Takao T, Shimonishi Y, Akino T, Yoshizawa T (1994) (1994) Effects of carboxyl methylation of photoreceptor G protein $\gamma$-subunit in visual transduction. J Biol Chem 269: 5163-5170. Medline

Furuta Y, Lagutin O, Hogan BL, Oliver GC (2000) Retina- and ventral forebrain-specific Cre recombinase activity in transgenic mice. Genesis 26:130-132. CrossRef Medline

Griggs AM, Hahne K, Hrycyna CA (2010) Functional oligomerization of the Saccharomyces cerevisiae isoprenylcysteine carboxyl methyltransferase, Ste14p. J Biol Chem 285:13380-13387. CrossRef Medline

Hrycyna CA, Sapperstein SK, Clarke S, Michaelis S (1991) The Saccharomyces cerevisiae STE14 gene encodes a methyltransferase that mediates C-terminal methylation of $\alpha$-factor and RAS proteins. EMBO J 10:1699_ 1709. Medline

Inglese J, Koch WJ, Caron MG, Lefkowitz RJ (1992) Isoprenylation in regulation of signal transduction by G-protein-coupled receptor kinases. Nature 359:147-150. CrossRef Medline

Kim E, Ambroziak P, Otto JC, Taylor B, Ashby M, Shannon K, Casey PJ, Young SG (1999) Disruption of the mouse Rcel gene results in defective Ras processing and mislocalization of Ras within cells. J Biol Chem 274: 8383-8390. CrossRef Medline

Kolandaivelu S, Chang B, Ramamurthy V (2011) Rod phosphodiesterase-6 (PDE6) catalytic subunits restore cone function in a mouse model lacking cone PDE6 catalytic subunit. J Biol Chem 286:33252-33259. CrossRef Medline

Kolandaivelu S, Singh RK, Ramamurthy V (2014) AIPL1, a protein linked to blindness, is essential for the stability of enzymes mediating cGMP metabolism in cone photoreceptor cells. Hum Mol Genet 23:1002-1012. CrossRef Medline

Kolesnikov AV, Rikimaru L, Hennig AK, Lukasiewicz PD, Fliesler SJ, Govardovskii VI, Kefalov VJ, Kisselev OG (2011) G-protein betagammacomplex is crucial for efficient signal amplification in vision. J Neurosci 31: 8067-8077. CrossRef Medline

Lobanova ES, Finkelstein S, Herrmann R, Chen YM, Kessler C, Michaud NA, Trieu LH, Strissel KJ, Burns ME, Arshavsky VY (2008) Transducin gamma-subunit sets expression levels of alpha- and beta-subunits and is crucial for rod viability. J Neurosci 28:3510-3520. CrossRef Medline

Michaelson D, Ali W, Chiu VK, Bergo M, Silletti J, Wright L, Young SG, Philips M (2005) Postprenylation CAAX processing is required for proper localization of Ras but not Rho GTPases. Mol Biol Cell 16:16061616. CrossRef Medline

Ohguro H, Fukada Y, Takao T, Shimonishi Y, Yoshizawa T, Akino T (1991) Carboxyl methylation and farnesylation of transducin gamma-subunit synergistically enhance its coupling with metarhodopsin II. EMBO J 10: 3669-3674. Medline

Ong OC, Ota IM, Clarke S, Fung BK (1989) The membrane binding domain of rod cGMP phosphodiesterase is posttranslationally modified by methyl esterification at a C-terminal cysteine. Proc Natl Acad Sci U S A 86:92389242. CrossRef Medline

Parish CA, Rando RR (1996) Isoprenylation/methylation of proteins enhances membrane association by a hydrophobic mechanism. Biochemistry 35:8473-8477. CrossRef Medline

Pérez-Sala D, Tan EW, Cañada FJ, Rando RR (1991) Methylation and demethylation reactions of guanine nucleotide-binding proteins of retinal rod outer segments. Proc Natl Acad Sci U S A 88:3043-3046. CrossRef Medline

Svensson AW, Casey PJ, Young SG, Bergo MO (2006) Genetic and pharmacologic analyses of the role of Icmt in Ras membrane association and function. Methods Enzymol 407:144-159. CrossRef Medline

Swanson RJ, Applebury ML (1983) Methylation of proteins in photoreceptor rod outer segments. J Biol Chem 258:10599-10605. Medline

Zhang H, Li S, Doan T, Rieke F, Detwiler PB, Frederick JM, Baehr W (2007) Deletion of PrBP $/ \delta$ impedes transport of GRK1 and PDE6 catalytic subunits to photoreceptor outer segments. Proc Natl Acad Sci U S A 104: 8857-8862. CrossRef Medline

Zhang H, Constantine R, Frederick JM, Baehr W (2012) The prenyl-binding protein $\operatorname{PrBP} / \delta$ : a chaperone participating in intracellular trafficking. Vision Res 75:19-25. CrossRef Medline 